\title{
LONG TERM EVOLUTION \\ OF SOLAR SECTOR STRUCTURE
}

\author{
LEIF SVALGAARD and JOHN M. WILCOX \\ Institute for Plasma Research, Stanford University, Stanford, Calif. 94305, U.S.A.
}

\begin{abstract}
The large-scale structure of the solar magnetic field during the past five sunspot cycles (representing by implication a much longer interval of time) has been investigated using the polarity (toward or away from the Sun) of the interplanetary magnetic field as inferred from polar geomagnetic observations. The polarity of the interplanetary magnetic field has previously been shown to be closely related to the polarity (into or out of the Sun) of the large-scale solar magnetic field. It appears that a solar structure with four sectors per rotation persisted through the past five sunspot cycles, with a synodic rotation period near 27.0 days, and a small relative westward drift during the first half of each sunspot cycle and a relative eastward drift during the second half of each cycle. Superposed on this four-sector structure there is another structure with inward field polarity, a width in solar longitude of about $100^{\circ}$ and a synodic rotation period of about 28 to 29 days. This 28.5 day structure is usually most prominent during a few years near sunspot maximum. Some preliminary comparisons of these observed solar structures with theoretical considerations are given.
\end{abstract}

Full text of the paper submitted to Solar Physics. 